\title{
Glucocorticoid-mediated inhibition of chemotherapy in ovarian carcinomas
}

\author{
CHENGWEN ZHANG $^{1 *}$, ALEXANDER MARMÉ $^{5,7^{*}}$, TILL WENGER $^{1}$, PAUL GUTWEIN $^{2,3}$, \\ LUTZ EDLER $^{8}$, WERNER RITTGEN ${ }^{8}$, KLAUS-MICHAEL DEBATIN ${ }^{9}$, PETER ALTEVOGT ${ }^{2}$, \\ JÜRGEN MATTERN ${ }^{1,4}$ and INGRID HERR ${ }^{1,6,9}$
}

\author{
${ }^{1}$ Molecular Urooncology, ${ }^{2}$ Tumour Immunology Programme, ${ }^{3}$ Department of Biostatistics, ${ }^{4}$ Cooperation Unit \\ Oncological Diagnostics and Therapy, German Cancer Research Center; Departments of ${ }^{5}$ Obstetrics and Gynaecology, \\ and ${ }^{6}$ Urology, University of Heidelberg, Heidelberg; ${ }^{7}$ Department of Gynaecology, University of Tuebingen; \\ ${ }^{8}$ Pharmacenter of the University Hospital Frankfurt; ${ }^{9}$ Department of Pediatrics, University of Ulm, Germany
}

Received August 17, 2005; Accepted October 3, 2005

\begin{abstract}
The glucocorticoid dexamethasone is frequently used as a co-treatment in cytotoxic cancer therapy, e.g. to prevent nausea, to protect normal tissue or for other reasons. While the potent pro-apoptotic properties and supportive effects of glucocorticoids to tumour therapy in lymphoid cells are well studied, the impact on the cytotoxic treatment of ovarian carcinoma is unknown. We tested apoptosisinduction, viability, tumour growth and protein expression using established cell lines, primary cell lines freshly isolated from patient material and a xenograft on nude mice. We found a general induction of resistance toward cytotoxic therapy by DEX-co-treatment in most of the examined ovarian cancer cells treated in vitro, ex vivo or in vivo. Resistance occured independently of cell density and was found at peak plasma levels of dexamethasone and below. Mechanistically, the dexamethasone-induced expression of survival genes may be involved in the resistance. These data show that glucocorticoid-induced resistance is common in ovarian carcinomas implicating that the use of glucocorticoids may be harmful for cancer patients.
\end{abstract}

\section{Introduction}

Ovarian cancer is still the gynaecological tumour with the highest mortality. This is usually partly due to the advanced

Correspondence to: Dr Ingrid Herr, German Cancer Research Center, Molecular Urooncology, Im Neuenheimer Feld 280, D-69120 Heidelberg, Germany

E-mail: i.herr@dkfz.de

${ }^{*}$ Contributed equally

Key words: cancer therapy, glucocorticoids, corticosteroids, nausea, apoptosis disease at the time of diagnosis but also due to the fact that most tumours become resistant to chemotherapy quickly. The primary therapy comprises cytoreductive surgery as well as adjuvant or neoadjuvant chemotherapy using schemes consisting of a platinum and a taxane derivative. Glucocorticoids (GCs) such as the synthetic corticoid, dexamethasone (DEX), are given as part of anti-emetic prophylaxis or the prevention of allergic reactions to taxanes (1). Newer agents, such as gemcitabine, are currently investigated in sequential therapy to avoid the development of drug resistance (2).

DEX and similar GCs were first introduced to tumour therapy on the basis of pro-apoptotic effects in lymphoid cells and on their effectiveness in treating tumour related edema, inflammation, pain, and electrolyte imbalance as well as stimulating the appetite and, most importantly, preventing the nausea and emesis caused by cytotoxic drugs (3-7). However, controlled randomized trials evaluating the potential impact of GCs on the growth of solid tumours and patient survival have never been performed. Concerns about the widespread use of GCs in the therapy of solid tumours have been expressed repeatedly $(8,9)$, involving studies showing enhanced percentages of metastases in breast cancer patients $(10,11)$ or an increased risk of skin cancer and non-Hodgkin lymphomas among users of systemic GCs (12). Recent studies suggest the inhibition of cytotoxic therapy-induced apoptosis by DEX in established lung, cervical and breast carcinoma cell lines in vitro, ex vivo and in vivo (13-15).

For the inhibition of apoptosis, a functional glucocorticoid receptor $(\mathrm{GR})$ is required $(13,14)$ and association with the transcriptional induction of serum and GC-inducible protein kinase-1 (SGK-1) and mitogen-activated protein kinase phosphatase-1 (MKP-1) has been suggested in human established breast cancer cell lines $(14,16)$. Thus, treatment with a GC alone or combined with chemotherapy led to an increased protein expression of SGK-1 and MKP-1 while specific inhibition of these molecules by small interfering RNA reversed the anti-apoptotic effects of GC treatment in breast cancer cells (14). Another putative link between apoptosis inhibition and the GR may be the anti-apoptotic and pro-proliferative protein, BAG-1, which has been shown to repress DNA 
binding of the GR in a process that requires prior binding of the chaperone, HSP-70 (17).

Since GCs are known to support cancer therapy in lymphoid tumour cells, the above results suggesting GC-induced resistance in some cells of solid tumours are surprising. Although these data might be important for patients, it is not known, whether GC-conferred resistance is a common or occasional problem of some types of solid tumour.

To analyze whether DEX might affect the outcome of cytotoxic therapy of ovarian carcinomas, established cell lines and tumour cells freshly isolated from patients were analysed. Treatment of the cells with cisplatin, gemcitabine or $\beta$-irradiation in the presence of DEX inhibited apoptosis and promoted proliferation in 13 of 14 ovarian carcinomas examined. The basal growth of xenografted tumours was enhanced in the presence of DEX and the therapeutic effect of cisplatin was inhibited. Under the same conditions, DEX enhanced the protein expression of SGK-1 and MKP-1 but not of BAG-1 in ovarian carcinoma cells.

\section{Materials and methods}

Ovarian carcinoma cell lines and culture. The ovarian carcinoma cell lines, SKOV3, OAW-42, OVM, and M130, are described (18). Cells were grown at $37^{\circ} \mathrm{C}$ in DMEM obtained from Life Technologies Gibco BRL (Karlsruhe, Germany), supplemented with $10 \%$ heat-inactivated fetal bovine serum (Sigma, Deisenhofen, Germany), $25 \mathrm{mM}$ HEPES and $2 \mathrm{mM}$ L-glutamine (all from Gibco/Life Technologies, Paisley, UK).

Isolation of fresh tumour cells. Solid tumours, peritoneal or pleural fluid specimens were obtained from patients who had histologically-confirmed epithelial ovarian carcinoma. Ovarian carcinoma cells from fluid specimens were isolated by density centrifugation using Ficoll separation solution (Biochrom, Berlin, Germany) at $400 \mathrm{~g}$ for $30 \mathrm{~min}$. The cells in the interface were collected and washed in phosphatebuffered saline. The preparation was visually inspected and found to contain between $70-80 \%$ carcinoma cells. The phenotype was also investigated by flow cytometry for Ep-CAM expressing cells using mAb HEA 125 (kind gift from Dr Gerd Moldenhauer, German Cancer Research Center, Heidelberg). A solid tumour from an ovarian carcinoma was minced in RPMI medium supplemented with $20 \%$ heat-inactivated fetal bovine serum (Sigma), $25 \mathrm{mM}$ HEPES, $2 \mathrm{mM}$ L-glutamine and Pen/Strep (all from Gibco/Life Technologies) under sterile conditions. Isolated cells were dissolved in RPMI medium supplemented with $20 \%$ FCS and immediately processed for MTT-analysis. Patient material was obtained under the approval of the ethics committee of the University of Heidelberg. Diagnoses were established by conventional clinical and histological criteria according to the World Health Organization (WHO).

Nude mice and xenografts. The tumour cells of patient 9 were established as a xenograft cell line (O4-9). Minced xenografts were injected subcutaneously into the right anterior flank of 6- to 10 -week old NMRI (nu/nu) female mice. After the tumours had reached a mean diameter of approximately 8$10 \mathrm{~mm}$, the mice were randomly divided into groups of 7 animals each and treatment was started. The mice were given $0.28 \mathrm{mg} / \mathrm{l} \mathrm{DEX}$ in the drinking water, and the daily amount of water consumed was approximately $30 \mathrm{ng} / \mathrm{g}$ body weight. Two days after adding DEX to the drinking water, therapy with cisplatin was started. Cisplatin $(5 \mathrm{mg} / \mathrm{kg})$ was injected on two consecutive days (days 2 and 3 ). Tumour growth was followed by measuring two diameters at days 3 and 4 with calipers and tumour volumes (v) were calculated using the formula, $v=1 / 2\left(\right.$ length $\mathrm{x}$ width ${ }^{2}$ ). Mice were humanely euthanized at tumour sizes $>3000 \mathrm{~mm}^{3}$.

Primary ovarian cancer cell line, MO68II. The primary ovarian cancer cell line, MO68II, was isolated from a peritoneal fluid specimen obtained from a patient with epithelial ovarian carcinoma and was used for experiments between passages 3 and 10 . The purity was $100 \%$ as confirmed by flow cytometry for Ep-CAM expressing cells using mAb HEA 125.

Drugs. A stock solution of cisplatin (Sigma) was prepared in DMSO at a concentration of $33 \mathrm{mM}$. Gemcitabine (kind gift from Eli Lilly, Indianapolis, IN, USA) was diluted in PBS to a $50 \mu \mathrm{M}$ stock. A $25 \mathrm{mM}$ stock of DEX (Sigma) was prepared in ethanol. Final concentrations of the solvents in medium were $0.1 \%$ or less. Cells were $\gamma$-irradiated in their flasks using a cesium $\gamma$-ray source.

Measurement of apoptosis. Cells were stained with fluoresceinthiocyanate (FITC)-conjugated annexin V (BD Biosciences, Heidelberg, Germany) and externalization of phosphatidylserine as well as the forward side scatter profile were identified by flow cytometry (FACScan, BD Biosciences) as described (13).

MTT-assay. Primary tumour cells were resuspended at $5 \times 10^{5} /$ $\mathrm{ml}$ in 96-well microplates, $100 \mu 1$ per well. After treatment, MTT-assay was performed as recently described (15).

Western blot analysis. Equal numbers of cells in each experimental condition were lysed with $2 \mathrm{X}$ Laemmli buffer and were fractionated on $10 \%$ SDS-PAGE gels. The fractionated proteins were transferred to nitrocellulose and were stained with Ponceau $\mathrm{S}$ dye to confirm equal protein loading. The membranes were then rinsed and incubated in the rabbit polyclonal antibodies, MKP-1, BAG-1 (both from Santa Cruz, Heidelberg, Germany), and SGK-1 (Stressgene, Victoria, Canada), or in the mouse monoclonal antibody, ACTIN (clone $\mathrm{C} 4$, ICN, Aurora, OH, USA). Bound antibodies were detected by anti-rabbit or anti-mouse/horseradish peroxidase conjugates (Santa Cruz) and an enhanced chemiluminescence system.

Statistical analysis. Each patient was investigated in a 2factorial design consisting of three doses of DEX $(0.1 \mu \mathrm{M}=$ DEX1, $1 \mu \mathrm{M}=\mathrm{DEX} 2,10 \mu \mathrm{M}=\mathrm{DEX} 3$ ) and a control as well as three doses of cytotoxic treatment and a control. Thus, there are a total of 16 experimental conditions. Viability of the cells under each condition was determined as mean of 3-8 replicates together with its standard deviation and then standardized on the result of the double control (no cytotoxic agent and no DEX applied); i.e. the viabilities of all conditions were divided by the mean of the double control. For each patient, 

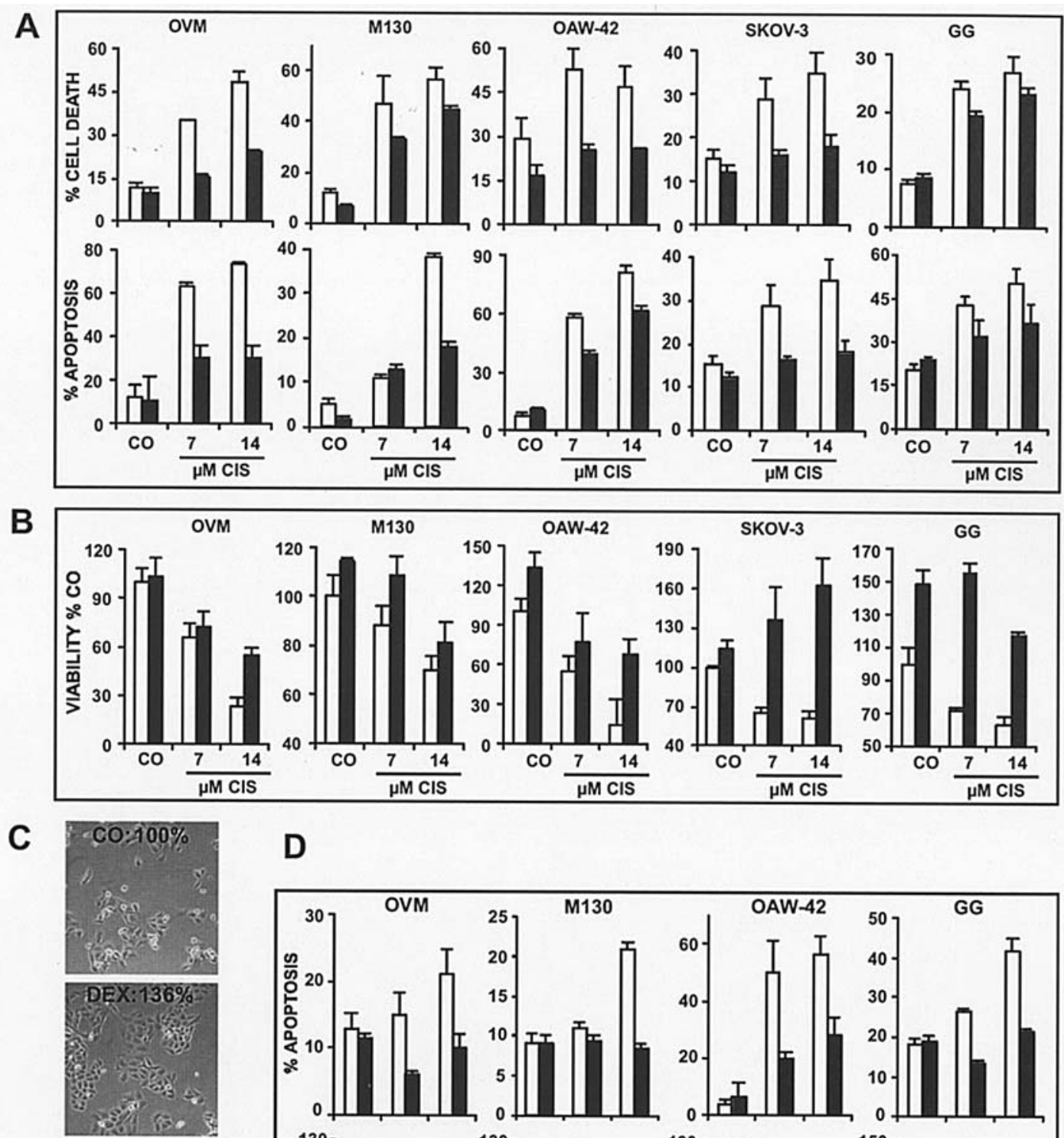

D
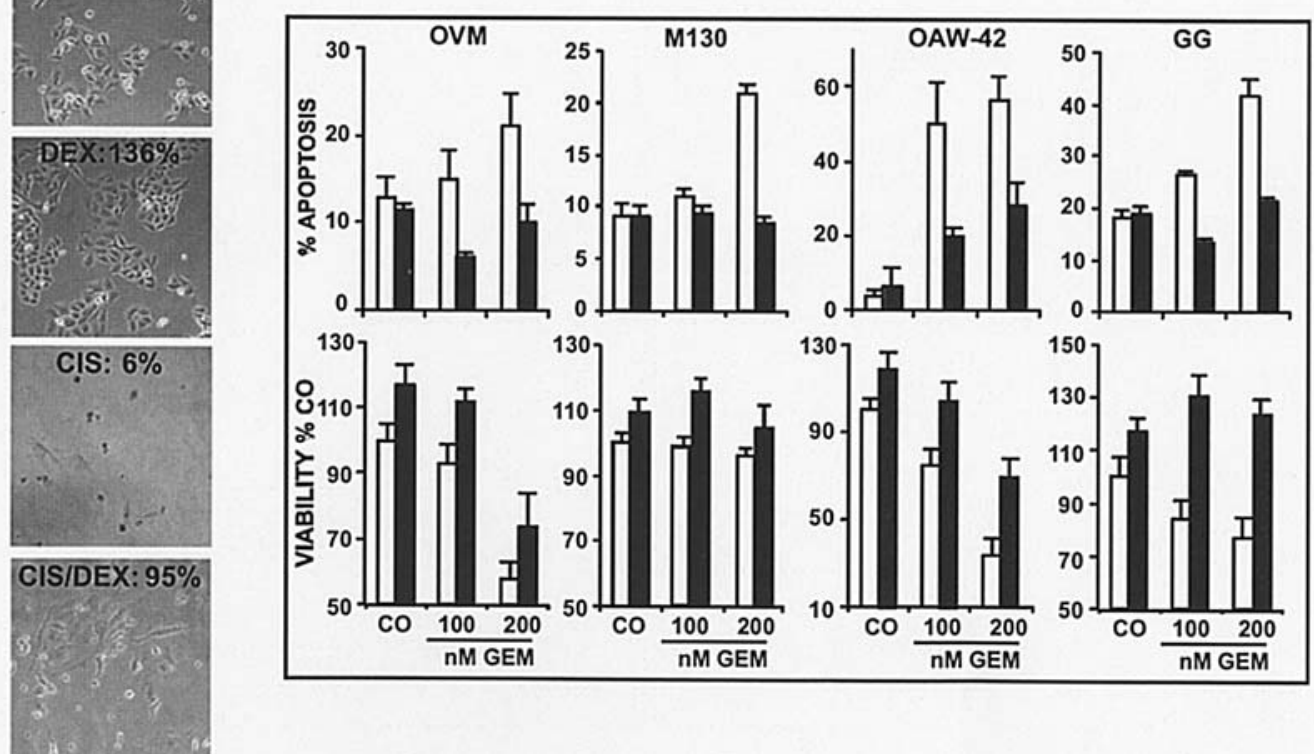

Figure 1. DEX inhibits cisplatin- and gemcitabine-induced apoptosis and promotes proliferation in vitro. OVM, M130, OAW-42, SKOV-3 and GG cells were left either untreated (CO) or were treated with cisplatin ( 7 or $14 \mu \mathrm{M}$ as indicated) in the absence (white bars) or presence (black bars) of DEX (1 $\mu \mathrm{M})$ which was added $48 \mathrm{~h}$ prior to cisplatin. Morphology (A) was analysed $48 \mathrm{~h}$ later by FACS-analysis using the FSC/SSC profile (\% Cell Death) and exposure of phosphatidylserine was detected by staining of the cells with annexin-FITC and FACS-analysis (\% apoptosis). (B) Viability was detected using MTT-assay. (C) OVM cells were treated as indicated $(0.1 \mu \mathrm{M} \mathrm{DEX,} 7 \mu \mathrm{M}$ CIS $)$. Two weeks later, the amount of viable cells was determined by trypan blue exclusion and cells were photographed. The percentage of viable cells is given in each picture. (D), OVM, M130, OAW-42 and GG cells were left either untreated (CO) or were treated with gemcitabine (100 and $200 \mathrm{nM}$ as indicated) in the absence (white bars) or presence (black bars) of DEX ( $1 \mu \mathrm{M})$ which was added $48 \mathrm{~h}$ prior to gemcitabine. Exposure of phosphatidylserine (A) was detected $48 \mathrm{~h}$ later by staining the cells with annexin-FITC and FACS-analysis and viability using MTT-assay. Experiments were performed three times with identical outcomes and standard deviations are shown.

the standardized means were compared separately for each therapeutic dose and for the control by comparing the three DEX doses with their respective control (cytotoxic treatment alone). Notice, that the four means (three DEX doses and control) under the condition of no therapy describe the effect of DEX alone while the other three sets of four means under the three doses of the therapeutic agent describe the resistance of the cells under treatment depending on DEX. 
Table I. Characteristics of tumour cells from patients 1-9 with ovarian cancer.

\begin{tabular}{lcccc}
\hline Patient & Histology & Pre-treatment & Origin of cells & DEX effect \\
\hline 1 & Serous cystadenocarcinoma & No & Ascites & Resistance \\
2 & Serous cystadenocarcinoma & Platinum & Pleural effusion & Resistance \\
3 & Serous cystadenocarcinoma & Platinum & Ascites & Resistance \\
4 & Papillary-serous cystadenocarcinoma & Platinum & Ascites & Resistance \\
5 & Papillary-serous cystadenocarcinoma & Platinum & Ascites & Resistance \\
6 & Papillary-serous cystadenocarcinoma & No & Primary tumour & Resistance \\
7 & Papillary-serous cystadenocarcinoma & Platinum & Ascites & Resistance \\
8 & Serous cystadenocarcinoma & Platinum & Primary cell line & Sensitivity \\
9 & Serous cystadenocarcinoma & No & Primary tumour & Resistance \\
\hline
\end{tabular}

We declared a DEX dose group resistant when its mean minus one standard deviation $\left(X_{I}-S D_{l}\right)$ was still larger than the mean plus one standard deviation $\left(X_{0}+S D_{0}\right)$ of the respective control group of that patient sample, $\mathrm{j}=1,2,3$ denoting the three dose groups receiving cytotoxic drugs. On this basis, scores were calculated per therapeutic dose (values $0,1,2,3$ ) as well as per patient in total (values ranging from 0 to 9 ). A patient was declared as being significantly resistant to cytotoxic treatment when he/she showed a score of 2 for at least one drug dose or when he/she reached the total score of 5 out of a maximum of 9 per time point (i.e. more than $50 \%$ ). The outcomes of a group of patients were summarized in respective ratios obtained by adding the individual scores for each condition as well as in total over the number of patients and by dividing through the number of patients. The results of the evaluation of each single patient are available upon request. The patient population was considered as suffering from resistance as a whole when, for one therapeutic dose, all ratios were higher than $50 \%$ or when 5 of the total of 9 combinations were higher than $50 \%$. This statistical method was applied independently to all three time points.

Xenografts on nude mice. A distribution free test for tumour growth curve analyses was used for therapy experiments with xenografted cancer cells as described (24).

\section{Results}

DEX prevents cisplatin-induced apoptosis and promotes proliferation in cell lines. To investigate whether DEX might protect ovarian carcinoma cell lines by interfering with apoptosis we treated OVM, M130, OAW-42, SKOV-3 and GG cells with cisplatin in the presence or absence of DEX. Cell death and apoptosis were examined $72 \mathrm{~h}$ later by FACSanalysis. While cisplatin alone strongly induced formation of apoptotic bodies indicating cell death as well as exposure of phospatidylserine as an early sign of apoptosis, the presence of DEX inhibited these effects in all cell lines examined (Fig. 1A). In light of the inhibited apoptosis we next analysed whether DEX might influence the growth of ovarian carcinoma cells. Cells were treated as described above and viability was detected by MTT-assay. While cisplatin alone strongly reduced proliferation, the presence of DEX neutralized the cytotoxic effect in all cell lines examined (Fig. 1B). Remarkably, DEX alone induced faster proliferation and reduced basal apoptosis. In order to know whether this protective effect of DEX might be long-lasting. we treated OVM cells with DEX in the presence or absence of cisplatin. Two weeks later, cells were photographed and viable cells were counted by trypan blue exclusion (Fig. 1C). DEX enhanced the basal proliferation from 100 to $136 \%$ and increased the viability of cisplatintreated cells from 6 to $95 \%$.

To ascertain whether DEX induces resistance towards other cytotoxic drugs, cells were treated with gemcitabine in the presence or absence of DEX. Apoptosis was analysed $48 \mathrm{~h}$ later by FACS-analysis. While gemcitabine alone strongly induced apoptosis and reduced proliferation, the presence of DEX prevented these effects (Fig. 1D). Thus, DEX inhibits apoptosis and promotes proliferation of cisplatin- and gemcitabine-treated ovarian carcinoma cells in vitro.

DEX induces therapy resistance in freshly isolated ovarian carcinomas. For a closer understanding of the clinical situation, we examined freshly isolated primary ovarian carcinoma cells. DEX was used in concentrations of 0.1-10 $\mu \mathrm{M}$ resembling the clinical setting $(5,19,20)$. Cells derived from patient 1 (for patient characteristics see Table I) were treated with cisplatin in the presence or absence of DEX. Cell death, apoptosis and viability were measured $48 \mathrm{~h}$ later. DEX prevented cell death as well as apoptosis and enhanced viability (Fig. 2A). DEXinduced cisplatin resistance was also found in tumour cells from five other patients as detected by the MTT-assay (Fig. 2B). Remarkably, tumour cells derived from two different pleural effusions from patient 2, obtained in a time interval of 6 weeks, exhibited DEX-induced resistance. Resistance in tumour cells of patients occured also after treatment with gemcitabine (Fig. 2C) or $\gamma$-irradiation (Fig. 2D). While DEX enhanced the basal proliferation of primary tumour cells derived from some patients, no effect or even repression was seen in tumour cells from others. Since this discrepancy might be due to high density culture conditions, we incubated primary cells from patient 3 at a 10x lower density of $10^{-4} / \mathrm{ml}$ and measured the viability compared to cells incubated at the usual density of $10^{-5} / \mathrm{ml}$. At $48 \mathrm{~h}$ after cytotoxic treatment, enhanced basal 


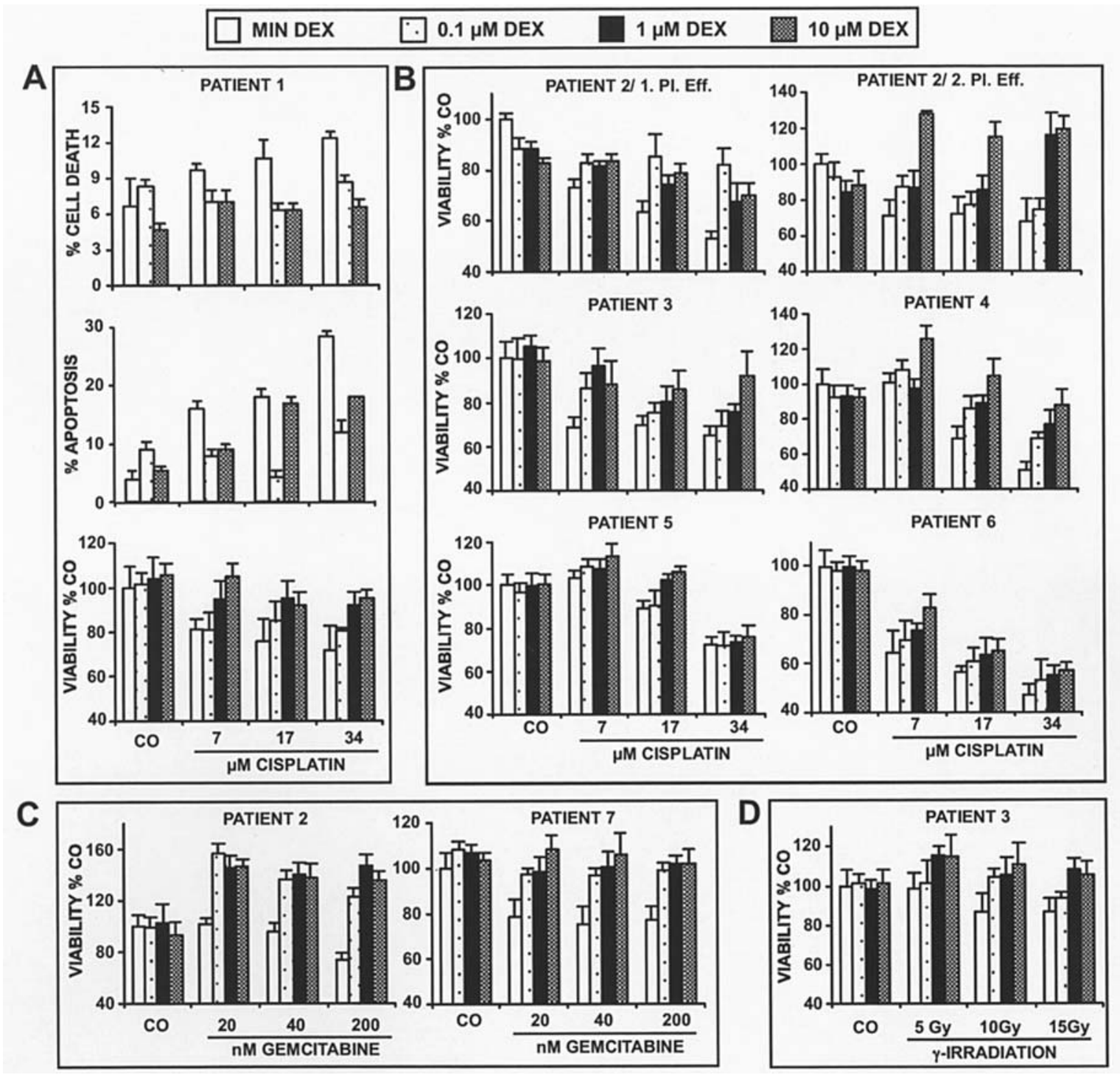

Figure 2. DEX generally inhibits cytotoxic therapy-induced apoptosis and promotes proliferation ex vivo. Tumour cells from patients with ovarian carcinoma were isolated and cultivated in 96-well plates at 5x105/ml. (A) As indicated, cells were left untreated (CO) or were treated with cisplatin (CIS) in the absence or presence of $\operatorname{DEX}(0.1,1$ or $10 \mu \mathrm{M})$ which was pre-incubated for $24 \mathrm{~h}$. Apoptosis, cell death and viability were measured $48 \mathrm{~h}$ after incubation with cisplatin. (B) Tumour cells isolated from patients 2-6 were treated as described above and viability was measured. From patient 2, tumour cells isolated from two different pleural effusions (1. Pl. Eff. and 2. Pl. Eff.) were examined. (C) Cells were cultured as described above but gemcitabine (20, 40, 200 nM) or (D) $\gamma$-irradiation $(5,20,15$ Gy) were used instead of cisplatin. Eight wells per treatment were analyzed and standard deviations are less than $10 \%$.

proliferation was found in cells incubated at the lower density but not in cells incubated at the higher density (Fig. 3A). However, at 72 or $96 \mathrm{~h}$, no significant enhancement of basal viability was found at either density. Also, DEX induced resistance toward cisplatin independently of cell density and time point. Therefore, a lower cell density may facilitate enhanced basal proliferation in response to DEX but does not influence DEX-induced therapy-resistance.

To examine the dose-response we diluted DEX to $0.0001 \mu \mathrm{M}$ and found a blocking of cytotoxic therapy below these peak plasma concentrations (Fig. 3B).

DEX does not enhance the acquired resistance of a heavily platinum pre-treated patient. In order to know whether DEX might further increase the resistance of cells which already acquired resistance from platinum pre-treatment we used primary tumour cells from patient 8 . This patient was heavily pre-treated with platinum which might be the reason for the high intrinsic resistance of the corresponding tumour cells as evident from a minimal effect of cisplatin on viability compared to tumour cells of other patients (Fig. 4A). In contrast to sensitive tumour cells, DEX co-treatment did not further enhance the resistance of tumour cells from patient 8 but rather sensitized them for the therapeutic effect of cisplatin (Fig. 4B).

DEX induces therapy resistance of primary ovarian carcinomas in vivo. To analyze the in vivo effect of DEX we xenografted tumour cells derived from a primary ovarian carcinoma from patient 9 to nude mice. Tumour volumes were measured on days three and four after the start of cisplatin therapy and were found to be reduced in mice treated with cisplatin alone (Fig. 5). However, DEX totally prevented the growth-inhibiting effect of cisplatin since the tumours grew as fast as tumours from untreated control mice. Also, DEX alone led to a faster basal growth of the xenografts. 

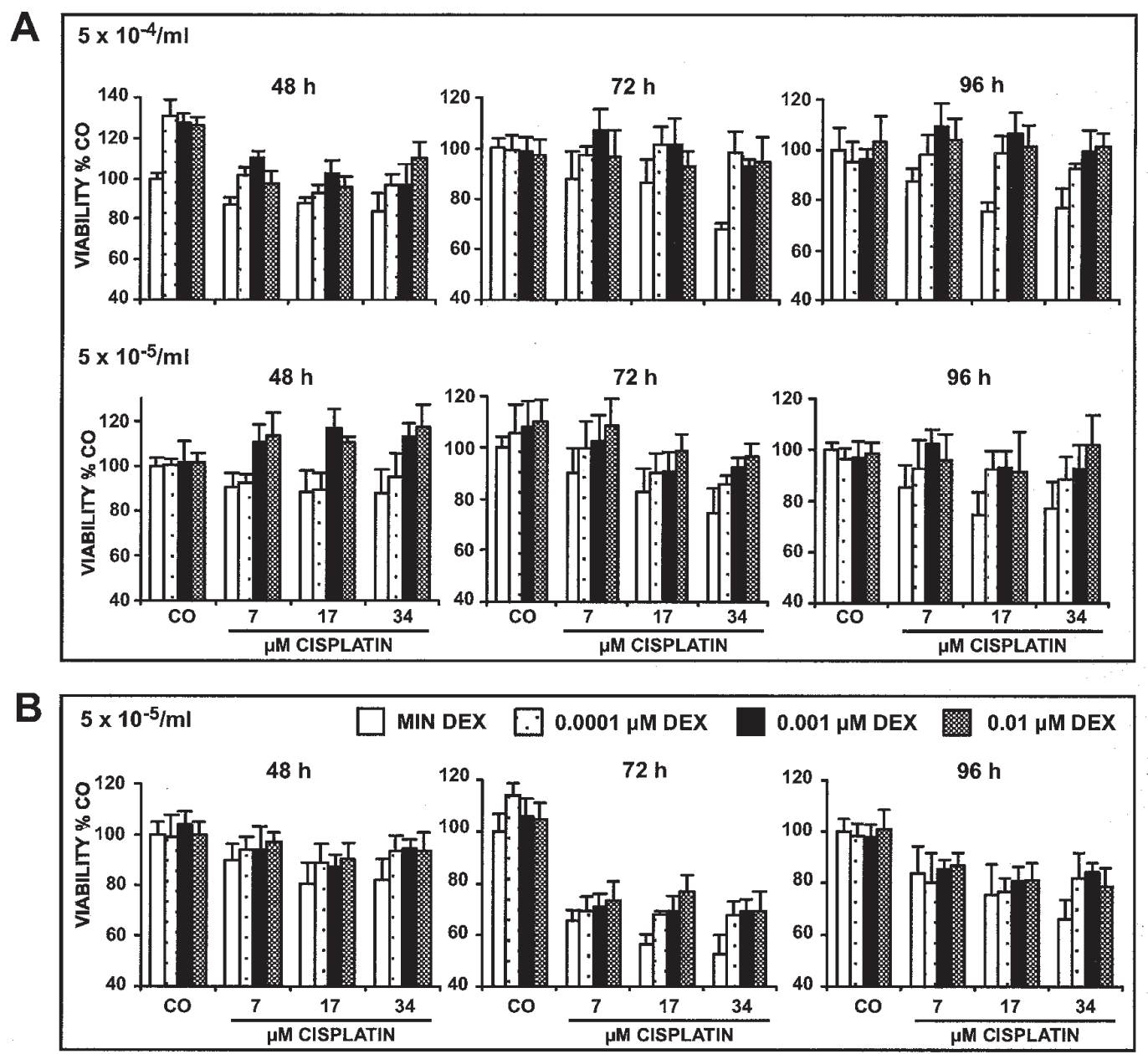

Figure 3. DEX induces resistance independent of cell density and below peak plasma levels. Ovarian carcinoma cells from patient 3 were cultivated at concentrations of (A) $5 \times 10^{4} / \mathrm{ml}$ and $5 \times 10^{5} / \mathrm{ml}$ in the absence or presence of DEX $(0.1,1$ or $10 \mu \mathrm{M})$ as described in Fig. 2. (B) Likewise, cells at a concentration of $5 \times 10^{5} / \mathrm{ml}$ were pre-incubated with DEX at concentrations below peak plasma levels $(0.01,0.001$ or $0.0001 \mu \mathrm{M})$ as indicated. $24 \mathrm{~h}$ after incubation with DEX, cisplatin was added. Viability was measured 24,48 or $72 \mathrm{~h}$ after incubation with cisplatin. Eight wells per treatment were analyzed and standard deviations are less than $10 \%$

A

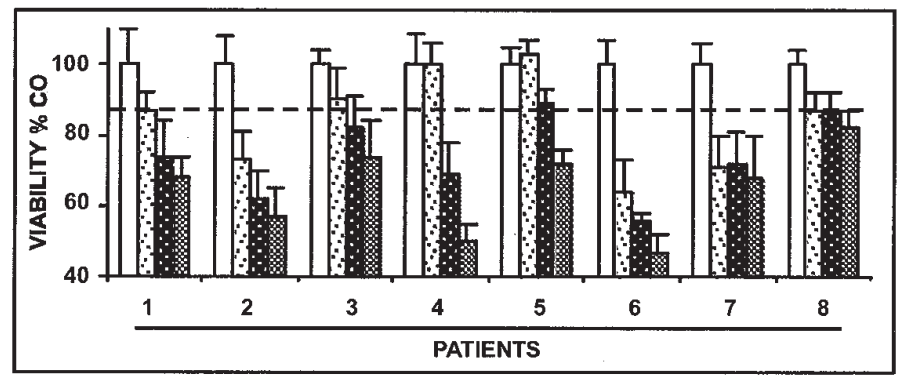

B

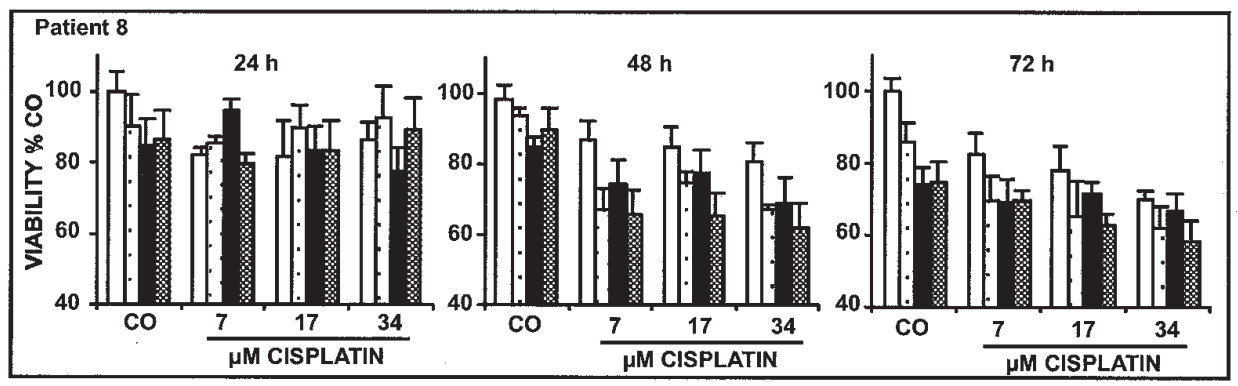

Figure. 4. DEX does not increase acquired resistance of tumour cells from a heavily platinum-pre-treated patient. (A) Viability of tumour cells derived from patients 1-8 was analyzed two days after treatment with cisplatin cisplatin alone which was used in the concentrations indicated. (B) Primary tumour cells from patient 8 were treated with DEX and cisplatin as described in Fig. 2, and 24, 48 or $72 \mathrm{~h}$ after treatment with cisplatin, the viability was determined. 


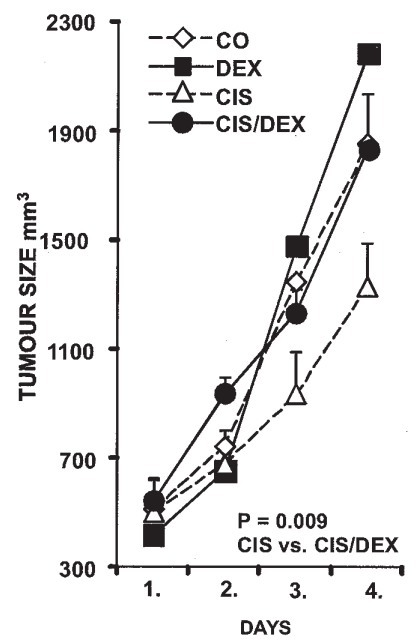

Figure 5. DEX inhibits therapy-induced carcinoma regression in vivo. O4-9 tumour cells derived from patient 9 were established as xenograft cell line and injected subcutaneously into nude mice treated as described in Materials and methods. The tumour volumes were measured daily after the start of therapy and the results are shown. Mice were humanely euthanized after the last measurement on day 4. Data are presented as mean of 7 animals and the single measurements are indicated. The P-values of 0.009 for CIS versus CIS/DEX and of 0.087 for CO versus CIS versus CIS/DEX versus DEX were determined according to the Koziol test for tumour growth curve analyses (24)

MKP-1 and SGK-1 but not BAG-1 may be involved in DEXinduced resistance. In a recent study, we demonstrated that DEX-co-treatment induces apoptosis resistance in lung and cervical carcinomas but not in leukemic $\mathrm{T}$ cells by cell type specific regulation of many apoptosis genes involving a functional GR (13). The upstream mechanism by which DEX inhibits apoptosis and promotes proliferation in ovarian carcinomas is totally unknown. Since enhanced expression of MKP-1 and SGK-1 is described to be involved in DEX-induced resistance in some human established breast cancer cell lines (14), we examined these molecules in GG ovarian carcinoma cells by Western blot analysis (Fig. 6A) and found enhanced levels of both proteins after treatment with DEX alone or with DEX and cisplatin together.

BAG-1 is another strong candidate known to link GRsignaling to impaired apoptosis and enhanced proliferation by interaction with HSP-70. Therefore, we examined changes in BAG-1 expression which might be induced by DEX. Using the cell lines, M130, GG and OAW-42, we did not detect any significant differences in expression of the three subunits of BAG-1 (p50, p36, p29) or of HSP-70 in any of the three examined cell lines (Fig. 6B). Therefore, MKP-1 and SGK-1 but not BAG-1 expression may be involved in the DEXinduced therapy resistance of ovarian carcinomas.

\section{Discussion}

Glucocorticoids (GCs) are known to exert pro-apoptotic and anti-proliferative effects in lymphoid cells. Hence, these agents are widely used as co-medication in cancer therapy including the highly effective synthetic GC, dexamethasone (DEX). Surprisingly, our data demonstrate the induction of resistance by DEX in ovarian carcinomas using concentrations resembling peak plasma levels found in patients and below.

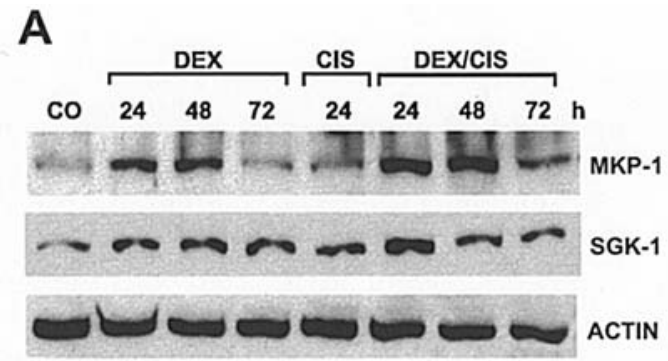

B

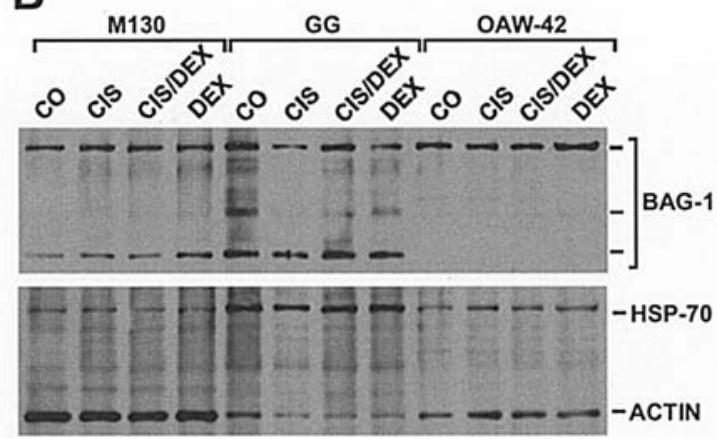

Figure 6. MKP-1 and SGK-1 but not BAG-1 may be involved in DEXinduced resistance. (A) The ovarian carcinoma cell line, GG, was treated with DEX $(1 \mu \mathrm{M})$ for 24,48 or $72 \mathrm{~h}$ as indicated. Cisplatin $(7 \mu \mathrm{M})$ was added to untreated cells (CIS) or to cells pre-incubated with DEX for $48 \mathrm{~h}$ (DEX/CIS). Proteins were harvested and analyzed $24 \mathrm{~h}$ after incubation with cisplatin by Western blot analysis using antibodies against MKP-1 (42 kDa) and SGK-1 (50-55 kDa). (B) The ovarian carcinoma cell lines, M130, GG and OAW-42, were treated as described in Fig. 1. Proteins were harvested $48 \mathrm{~h}$ later. Western blot analysis using antibodies against BAG-1 and HSP70 were performed. The p50, p36 and p29 subunits of BAG-1 as well as the band of HSP-70 $(70 \mathrm{kDa})$ are indicated. ACTIN (44 kDa) expression is a marker for equal loading. The experiments were performed three times with a similar outcome.

Since lower concentrations occur in vivo at later times after DEX administration, as plasma levels decline, this point leads to the question of whether the level of naturally occurring GCs may interfere with cancer therapy. The latter issue is addressed by our results using a xenografted ovarian carcinoma clearly suggesting that exogenously added DEX stimulates the growth of tumours in vivo well above levels originating from endogenous GCs. Since pharmacokinetic studies show that peak plasma levels in response to an anti-emetic dose of DEX were maintained for several hours and drop down very slowly (20), clearance of GCs and, thereby, resistance may last for a long time in patients.

We further demonstrate that the blocking effect of DEX is not specific to any individual anticancer drug but seems to be a more common phenomenon since it occurred with cisplatin-, gemcitabine- and $\gamma$-irradiation. Our results obtained with ovarian carcinomas may be transferred to other solid tumours, since we have found, in our ongoing screening project, induction of resistance by GCs in $95 \%$ of 161 examined fresh surgical specimens, xenografts on mice and established cell lines of tumours including bladder, bone, brain, breast, cervix, colon, liver, lung, kidney, ovary, pancreas, prostate, rectum and testis, together with neuroblastomas, and melanomas (15 and our unpublished data).

While our in vitro cell culture data are quite convincing, the data obtained with patient material are less consistent since 
DEX did not enhance basal proliferation in each carcinoma but even repressed it in some tumour cells. Also, DEX sensitized tumour cells derived from one patient exhibiting high intrinsic resistance toward cisplatin therapy. We actually do not know the reason for these variations in vivo, but assume that they might be due to intrinsic properties of the tumours, pretreatment of the patients and the quality of preparations of ovarian carcinoma cells from ascites or pleural effusion. Thus, the purity of isolated ovarian carcinoma cells differed between $70-80 \%$. However, the present results are important for the management of therapy. Our findings strongly suggest that GCs are able to induce resistance to cytotoxic therapy in clinical settings, specifically in patients with ovarian cancer. This point was recently addressed in a retrospective clinical study evaluating the records of 245 of a total of 763 patients with ovarian carcinoma and no negative outcome of glucocorticoid treatment on the survival of patients was found (21). However, this study cannot give a definitive answer to the question of whether GC treatment, as part of an anti-emetic regimen, prevention of allergic reactions, or as immunosuppressive therapy, is safe in patients with ovarian carcinoma - the reasons are discussed elsewhere (22). Furthermore, there are other clinical examinations which clearly show a negative impact of GCs, e.g. an increased metastatic potential in breast cancer patients and an enhanced risk of skin cancer and lymphomas among users of systemic GCs (10-12).

The mechanisms by which GCs induce apoptosis in lymphoid cells are well studied. These include depolarization of the mitochondrial membrane potential, enhanced expression of the death receptor, CD95, and its ligand, followed by activation of the caspase cascade (23). The same signaling cascades which are induced in lymphoid cells are blocked in carcinoma cells by GCs, thereby inhibiting chemo- and radiation therapy-induced apoptosis (13). Our present data together with recently published data obtained in human established breast cancer cell lines (14) suggest the involvement of MKP-1 and SGK-1 but not of BAG-1 in the upstream regulation of these events in ovarian carcinoma cell lines.

In conclusion, we have shown that the application of DEX renders ovarian cancer cells resistant to cytotoxic therapy. While the anti-emetic effect and protection of normal tissue by GCs may be of benefit to patients, the counteracting of cytotoxic treatment may minimize the growth retardation of tumours by cytotoxic treatment. Re-evaluation of patient files and controlled randomized prospective clinical studies are urgently needed.

\section{Acknowledgements}

We thank Dr G. Moldenhauer for helpful discussion and M. Mildenberger for excellent technical assistance.

\section{References}

1. Natarajan M, Saravanan SM and Elson DL: Advanced ovarian carcinoma as a chronic disease: a case report and review. S D J Med 56: 515-521, 2003.
2. Kaye SB: Chemotherapy for ovarian cancer: yesterday, today and tomorrow. Br J Cancer 89: 51-52, 2003.

3. Cheng L, Du C, Murray D, et al: A GFP reporter system to assess gene transfer and expression in human hematopoietic progenitor cells. Gene Ther 4: 1013-1022, 1997.

4. Aapro MS: Present role of corticosteroids as antiemetics. In: Recent Results in Cancer Research. Springer Verlag, Berlin, pp91-100, 1991.

5. The Italian Group for Antiemetic Research: Dexamethasone, granisetron, or both for the prevention of nausea and vomiting during chemotherapy for cancer. N Engl J Med 332: 1-5, 1995.

6. The Italian Group for Antiemetic Research: Dexamethasone alone or in combination with ondansetron for the prevention of delayed nausea and vomiting induced by chemotherapy. N Engl J Med 342: 1554-1559, 2000

7. Kirkbride P, Bezjak A, Pater J, et al: Dexamethasone for the prophylaxis of radiation-induced emesis: a National Cancer Institute of Canada Clinical Trials Group phase III study. J Clin Oncol 18: 1960-1966, 2000.

8. Haid M: Steroid antiemesis may be harmful. N Engl J Med 304: 1237, 1981.

9. Rutz HP: Effects of corticosteroid use on treatment of solid tumours. Lancet 360: 1969-1970, 2002.

10. Iversen HG and Hjort GH: The influence of corticoid steroids on the frequency of spleen metastases in patients with breast cancer. Acta Pathol Microbiol Scand 44: 205-212, 1958.

11. Sherlock P and Hartmann WH: Adrenal steroids and the pattern of mestastases of breast cancer. JAMA 181: 313-317, 1962.

12. Sorensen HT, Mellemkjaer L, Nielsen GL, Baron JA, Olsen JH and Karagas MR: Skin cancers and non-Hodgkin lymphoma among users of systemic glucocorticoids: a population-based cohort study. J Natl Cancer Inst 96: 709-711, 2004.

13. Herr I, Ucur E, Herzer K, et al: Glucocorticoid cotreatment induces apoptosis resistance toward cancer therapy in carcinomas. Cancer Res 63: 3112-3120, 2003.

14. Wu W, Chaudhuri S, Brickley DR, Pang D, Karrison T and Conzen SD: Microarray analysis reveals glucocorticoid-regulated survival genes that are associated with inhibition of apoptosis in breast epithelial cells. Cancer Res 64: 1757-1764, 2004.

15. Gassler N, Zhang C, Schnabel PA, et al: Dexamethasoneinduced cisplatin and gemcitabine resistance in lung carcinoma samples treated ex vivo. Br J Cancer 92: 1084-1088, 2005.

16. Wu W, Pew T, Zou M, Pang D and Conzen SD: Glucocorticoid receptor-induced MKP-1 expression inhibits paclitaxel-associated MAP kinase activation and contributes to breast cancer cell survival. J Biol Chem 280: 4117-4124, 2005.

17. Cato AC and Mink S: BAG-1 family of cochaperones in the modulation of nuclear receptor action. J Steroid Biochem Mol Biol 78: 379-388, 2001.

18. Gast D, Riedle S, Schabath H, et al: L1 augments cell migration and tumor growth but not $B 3$ integrin expression in ovarian carcinomas. Int J Cancer 115: 658-665, 2005.

19. Ioannidis JP, Hesketh PJ and Lau J: Contribution of dexamethasone to control of chemotherapy-induced nausea and vomiting: a meta-analysis of randomized evidence. J Clin Oncol 18: 3409-3422, 2000.

20. Brady ME, Sartiano GP, Rosenblum SL, Zaglama NE and Bauguess CT: The pharmacokinetics of single high doses of dexamethasone in cancer patients. Eur J Clin Pharmacol 32: 593-596, 1987.

21. Muenstedt K, Borces D, Bohlmann MK, Zygmunt M and von Georgi R: Glucocorticoid administration in antiemetic therapy. Cancer 101: 1696-1702, 2004.

22. Rutz HP and Herr I: Glucocorticoid administration in antiemetic therapy: is it safe? Cancer 103: 2696, 2005.

23. Schmidt S, Rainer J, Ploner C, Presul E, Riml S and Kofler R: Glucocorticoid-induced apoptosis and glucocorticoid resistance: molecular mechanisms and clinical relevance. Cell Death Differ 11 (suppl 1): S45-S55, 2004.

24. Koziol JA, Maxwell DA, Fukushima M, Colmerauer ME and Pilch YH: A distribution-free test for tumor growth curve analyses with application to an animal tumor immunotherapy experiment. Biometrics 37: 383-390, 1981. 